Research Article

\title{
Prevalence of Dental Caries and Its Associated Factors among Primary School Children in Ethiopia
}

\author{
Anguach Shitie $\mathbb{D}^{1},{ }^{1}$ Rahel Addis, ${ }^{2}$ Abebe Tilahun, ${ }^{3}$ and Wassie Negash ${ }^{2}$ \\ ${ }^{1}$ College of Medicine and Health Sciences, Wollo University, Dessie, Ethiopia \\ ${ }^{2}$ College of Medicine and Health Sciences, Debre Berhan University, Debreberhan, Ethiopia \\ ${ }^{3}$ College of Medicine and Health Sciences, Haramaya University, Harar, Ethiopia \\ Correspondence should be addressed to Anguach Shitie; anguachshitie20@gmail.com
}

Received 12 December 2020; Revised 15 February 2021; Accepted 5 March 2021; Published 15 March 2021

Academic Editor: Alessandro Leite Cavalcanti

Copyright (c) 2021 Anguach Shitie et al. This is an open access article distributed under the Creative Commons Attribution License, which permits unrestricted use, distribution, and reproduction in any medium, provided the original work is properly cited.

Background. Dental caries (decay or cavities) is a breakdown of teeth as a result of bacteria. Dental caries is one of the preventable oral health problems and the most common chronic diseases in childhood. Poor dental and oral health affect the quality of children's life. Objectives. To estimate the prevalence of dental caries and its associated factors among primary school children in Alem Ketema, North Showa, Ethiopia. Methodology. An institution-based quantitative cross-sectional study was conducted on 422 primary school children, from February 9 to March 8, 2020. Pretested structured questionnaire and oral examinations were conducted by using the WHO criteria (DMFT) index to collect the data. The data were entered into Epi-Data 3.1 software, and analysis was carried out using SPSS version 23 and variables with $P$ value $\leq 0.05$ in the multivariable binary logistic analysis were considered as a cut-point to declare statistical significance. Result. The prevalence of dental caries was 46.9\% (95\% CI: 42.1, 51.7). The mean Decayed, Missing, and Filled Teeth (DMFT) index was $1.28( \pm 1.21)$. Being male $(\mathrm{AOR}=1.975,95 \% \mathrm{CI}=1.067-3.66)$, lack of parents' insistence $(\mathrm{AOR}=2.052,95 \% \mathrm{CI}=1.079-3.902)$, lack of oral health education program $(\mathrm{AOR}=4.753,95 \%$ $\mathrm{CI}=1.69-13.38)$, history of dental aches $(\mathrm{AOR}=2.88,95 \% \mathrm{CI}=1.55-5.32)$, and poor habit of mouth wash $(\mathrm{AOR}=4.74,95 \%$ $\mathrm{CI}=2.55-8.79$ ) were found significantly associated with dental caries. Conclusion. The prevalence of dental caries was higher and a common public health problem among school children. Lack of parent's insistence, sex, dental ache history, poor practices of mouth wash, and lack of oral health education were significant predictors of dental caries. Therefore, oral health education program and parents encouraging their children to practice oral hygiene are important to prevent dental caries.

\section{Introduction}

According to the World health organization (WHO), dental caries (tooth decay) is defined as the destruction of the enamel layer of the tooth by acids produced by the action of bacteria on sugar [1]. It is widely known that dental caries is one of the preventable oral health problems and the most common chronic infectious, childhood diseases [2]. Dental caries is highly prevalent and has a negative impact on children's quality of life, and is a major public health problem in the whole world [3].

It is estimated that nearly 3.5 billion people are affected by oral disease in the world [4]. Approximately 2.4 billion or $36 \%$ of the world population have dental caries in their permanent teeth [5]. More than 530 million of children lose their primary teeth due to dental caries [4]. Due to lack of health education and insufficient preventive measures, there is a high prevalence of morbidity that highly affects the health status of children [6]. In the United States, dental caries is the most common chronic childhood disease. It is five times more common than asthma [5]. In England, over 60,000 children aged $0-19$ years were admitted to hospital to have teeth removal under a general anesthesia [7]. Dental caries remains highly prevalent in most of the developing countries [8].

Dental caries results from a complex interaction of factors like host susceptibility, bacteria, diet, and time (duration). The bacteria and sugary food act together to form 
acid productions that result in the formation of teeth cavitation [9]. Consequently, the acid destroys the enamel surface; if the process is not seen, it will result in progressive destruction of the tooth [10]. Dental caries is highly increasing among children due to the excessive consumption of sugary substances, poor oral hygiene, lack of fluoride exposure, and inadequate health service utilization $[11,12]$. Poor oral health can lead to tooth decay which affects the growth and maturation of secondary dentition (permanent dentition) which leads to malocclusion of teeth [9]. Dental caries has many complications like toothache, pulpitis, tooth loss, dental discoloration, and Ludwig angina [5].

Globally, it has been estimated that $60 \%$ to $90 \%$ of school children have dental caries; this means that six to nine children in every ten are affected by tooth decay [13]. Oral health affects both physically and psychologically [14]. Children with dental caries have difficulties in sleeping, playing, eating, performing academic activity and communication problems due to missing, discolored, or damaging teeth. Generally, it affects children's physical growth, self-esteem, and social development [15].

Dental caries has a high financial burden on parents of affected children [16]. In high-income countries, dental treatment is costly with $5 \%$ of the total health expenditure and $20 \%$ of out-of-pocket health expenditure [4]. The burden of oral health in the United States is annually over 20 million working days and 51 million school hours are lost alone due to oral disease and its treatment [17]. In low- and middle-income countries, the incidence of dental caries is rapidly increasing among children and adults [18]. Oral health impacts general health, dental infection increases risk of pneumonia, gum disease can complicate diabetes, and oral bacteria are associated with infective arthritis and associated with endocarditis inflammation of the heart's inner lining [19]. A healthy mouth not only enables the nutrition of the physical body, but also enhances social interaction and promotes self-esteem and feelings of well-being [20, 21].

Schools have a major role in promoting and implementing health problems like dental caries. Schools are the best center for effectively implementing the comprehensive healthcare program as children are easily accessible at school [22]. In Ethiopia, oral health problems, prevention, and treatment get inadequate attention in the government so the identification of prevalence and risk factors will help to suggest interventions in order to reduce dental caries.

\section{Objectives}

The aim of this study is to assess the prevalence of dental caries and associated factor among primary school children in Alem Ketema, North Showa, Ethiopia, 2020.

\section{Methodology}

3.1. Study Area and Period. The study was conducted in Alem Ketema town, which is located in North Showa Zone, Amhara region, and has a total population of 33,669 . It is located 180 kilometers away from the capital city of Addis Ababa and 142 kilometers away from Debre Berhan city, at an elevation of 1,885 meters. Alem Ketema has 2 primary schools, one secondary school, and one preparatory school. It also has one district hospital, one health center, and three private clinics and three pharmacies. The study was conducted from February to March 2020.

3.2. Sampling Technique. The school was selected by simple random sampling technique. Study participants were selected using a systematic random sampling technique with every interval of 5 from the student list using the formula $K=N / n$. The first student was selected using lottery method.

3.3. Study Population. Primary school children in the selected primary school were the study population.

3.4. Inclusion Criteria. All primary school children who were attending class during the data collection period, and their parents were included in this study.

3.5. Sample Size Determination. The sample size was calculated using a single population proportion formula by considering the following assumptions, a prevalence of $48.5 \%$ of dental caries in primary school children based on a similar study done in Finote Selam Town primary school [9], $95 \%$ confidence level, and marginal of error 5\%:

$$
\begin{aligned}
& n=\frac{z a^{2}}{2 p} \frac{(1-p)}{d^{2}} \\
& n=(1.96)^{2} \times 0.485 \frac{(1-0.485)}{0.05^{2}}, \\
& n=383
\end{aligned}
$$

where level of significance $=5 \% \quad(0.05) \quad$ and nonresponse $=10 \%, n=422$.

Finally, $10 \%$ non-response rate was added to the calculated sample size.

For the second objective, the sample size for associated factors was determined using Epi info version 7 at confidence interval $(1-\alpha)=95 \%$ and power $(1-\beta)$ of $80 \%$ was calculated by considering factors like grade level, habit of cleaning teeth, and consumption of sweet food. Finally, the calculated sample size for independent factors was smaller than the prevalence. So, the largest one, the prevalence sample size 422, was taken (Table 1).

3.6. Data Collection Tools, Method, and Data Quality Assurance. The data were collected using a pretested structured questionnaire from student's parents or caregiver. Data were collected by face-to-face interviews by two trained female diploma nurses. The data collectors were selected based on familiarity with the study area and local language. Two days of training was given, and pretest was done on five percent of the sample, to assess the clarity of the question, their competence, and understanding of the data collector. Double data entry was 
TABLE 1: Sample size calculation for research on dental caries among primary school children, Ethiopia, 2020.

\begin{tabular}{lccccc}
\hline \multirow{2}{*}{ Associated factors } & Reference & \multicolumn{5}{c}{ caries (\%) } & AOR & Sample size \\
& & Yes & No & & \\
\hline Grade-level & {$[23]$} & 31.9 & 12.2 & 3.9 & 162 \\
Cleaning teeth & {$[23]$} & 16 & 36.6 & 2.6 & 195 \\
Consumption of sugar & {$[9]$} & 50.4 & 17.6 & 3.41 & 100 \\
\hline
\end{tabular}

done to maintain the quality and consistency of data. Entry errors were checked and corrected by going back to the questionnaires.

3.7. Oral Examination. Clinical examinations for dental caries were performed by one dentist. Dental examination was performed on each child by using a disposable glove, daylight, dental mirror, and wooden spatula. The dental caries diagnosis protocol was obtained from WHO dental caries diagnosis guideline, by using the Decayed, Missing, and Filled Teeth (DMFT) index which is one of the most widely used methods in oral health for assessing dental caries prevalence.

\subsection{Measurements}

DMFT: the sum of the number of decayed (D), missing due to caries $(\mathrm{M})$, and filled $(\mathrm{F})$ teeth in the permanent teeth

Mean DMFT: the sum of individual DMFT values divided by the sum of the population or examined population

Dental aches: pain or inflammation in or around the tooth, often caused by tooth decay or infection

3.9. Statistical Analysis. The data were checked for its completeness and were entered using Epi-Data 3.1 and exported to SPSS software and analysis was carried out using statistical package for social sciences (SPSS) version 23. The data were summarized and organized using tables, texts, and chat. The reliability test was done with a Cronbach Alpha test, and it was 0.75 . Variables with $95 \% \mathrm{CI}$ and $P$ value $<0.25$ during the bivariate analysis were entered into multivariable analysis. Multi-co-linearity test was carried out to see the correlation between independent variables using a multi-colinearity coefficient and by variance inflation factor (VIF). Hosmer-Lemeshow was used to test model fitness (0.94). Finally, variables with $P$ value less than or equal to 0.05 in multivariable analysis were considered significant.

\section{Results}

4.1. Sociodemographic Characteristics. A total of 422 children participated in this study which gives a response rate of $100 \%$. The mean age of the children was $10.9(\mathrm{SD} \pm 2.3)$. More than half of children 242 (57.3\%) were aged 11-14. Around two hundred twenty-three (52.8\%) of the children were grades 5-8. About two hundred forty-six (58.3\%) of the respondents were male (Table 2 ).

4.2. Dietary- and Oral Hygiene-Related Characteristics. From the total children, $365(86.5 \%)$ consumed sweet food, of whom $114(31.1 \%)$ consumed daily and 144 (34.1\%) used bottled food during childhood period. One hundred sixtyfive $(39.1 \%)$ of children were practicing tooth brush. The majority of the children, 355 (84.1\%), did not use toothpaste. From the total children, one hundred twenty-four $(29.4 \%)$ of parents insisted that their children clean their teeth. One hundred sixty-nine (40.0\%) of children have experienced dental ache (Table 3).

4.3. Prevalence of Dental Caries. The prevalence of dental caries in this study was 198 (46.9\%) with 95\% CI (42.1-51.7). The mean Decay, Missing, and Filled Teeth (DMFT) index was $1.28( \pm 1.21)$ among the study participants; none of them had filled treatment (Figure 1).

4.4. Factors Associated with Dental Caries. Bivariable and multivariable logistic regression analysis was carried out to determine the association between independent variables and dental caries. Hence, in the bivariable analysis, sex, age, grade level, consumption of sweet food, habit of brush teeth, history of dental aches, oral health education, lack of parent insistence, and poor mouth wash after food were associated with dental caries whereas. On multivariable logistic regression, sex, history of dental aches, lack of oral health education, lack of parents' insistence, and poor mouth wash after food were the independent predictors of dental caries.

In this study, male children were 2 times more likely to have dental caries than females $(A O R=1.97 \quad 95 \%$ $\mathrm{CI}=1.067-3.66)$. Children who had a history of dental aches were 3 times more likely to have dental caries $(\mathrm{AOR}=2.88$ $95 \% \mathrm{CI}=1.55-5.32)$. Children with lack of oral health education were 4.7 times more likely to have dental caries ( $\mathrm{AOR}=4.753$ 95\% CI = 1.69-13.38). Children who had poor mouth wash after food were 4.7 times more likely to develop dental caries than children who practiced mouth wash after food $(\mathrm{AOR}=4.7495 \% \mathrm{CI}=2.55-8.79)$. Children whose parents lacked insistence were 2 times more likely to develop dental caries $(\mathrm{AOR}=2.05295 \% \mathrm{CI}=1.079-3.902)$ (Table 4).

\section{Discussion}

This study tried to assess the prevalence of dental caries and its associated factors among primary school children. Based on the findings, the prevalence of dental caries in this study was $46.9 \%$ (95\% CI 42.1, 51.7). This is consistent with study done in Finote Selam and Chennai (India) [8, 24]. The prevalence of dental caries in this study was relatively higher than a study done in Aksum (35.4\%) and Bahir Dar (21.8\%) $[11,25]$ but was lower than a study conducted in Najran, Saudi Arabia (71.5\%), Eritrea (78\%), Tamil Nadu (63.9\%), and Brazil (55.5\%) [26-29]. This difference might be due to 
TABLE 2: Sociodemographic characteristics of primary school children in Alem Ketema, North Shewa, Ethiopia, $2020(n=422)$.

\begin{tabular}{lc}
\hline Variables & Frequency $N(\%)$ \\
\hline Age & \\
$7-10$ & $180(42.7)$ \\
$11-14$ & $242(57.3)$ \\
Sex & \\
Male & $246(58.3)$ \\
Female & $176(41.7)$ \\
Residency & \\
Urban & $416(98.6)$ \\
Rural & $6(1.4)$ \\
Grade level & \\
$1-4$ & $199(47.2)$ \\
5-8 & $223(52.8)$ \\
Religion & \\
Orthodox & $415(98.3)$ \\
Catholic & $7(1.7)$ \\
Mother's education level & \\
Illiterate & $168(39.8)$ \\
Can read and write & $106(25.1)$ \\
Grade 1-8 & $64(15.2)$ \\
Grade 9-12 & $50(11.8)$ \\
Above grade 12 & $34(8.1)$ \\
Father's education level & \\
Illiterate & $56(13.3)$ \\
Can read and write & $127(30.1)$ \\
Grade 1-8 & $96(22.7)$ \\
Grade 9-12 & $92(21.8)$ \\
Above grade 12 & $51(12.1)$ \\
\hline
\end{tabular}

sample size difference and sociodemographic differences like age category and sex.

In this study, the mean DMFT was 1.28 which was higher compared to study done in Ferozepur (India) [23], Finote Selam [23], and Nigeria [24, 30]. This might be due to poor oral hygiene practice and dietary habits. However, the value was lower compared to a study done in Iran [31], Eritrea [27], and Mumbai (India) [22]. It might be due to that filled teeth might be missed during examination.

Sex of the children was one of the factors significantly associated with dental caries in this area. Males were 2 times more likely to have dental caries than female. This finding was similar to a study conducted in Bihar (India) [32] and Nigeria [30]. The reason could be due to the difference in sex composition of the sample. In contrast to this study, in Iran females were 1.4 times more likely to have dental caries [33]. This may be due to lower salivary flow rate, tooth eruption, and females acquiring their teeth earlier than males [34].

This study found that children with dental aches were 3 times more likely to have dental caries; similarly findings were obtained in Bahar Dar where children with dental aches were 6.3 times more likely to have dental caries [11] and a study conducted in Aksum showed that children who had dental pain were 1.8 times more likely to have dental caries [25]. This might be due to the fact that the dental aches may be associated with poor habit of tooth cleaning and exposure to sweet drinks and food.
TABLE 3: Dietary- and oral hygiene-related characteristics of Alem Ketema Primary School children, North Shewa, Ethiopia, 2020 $(n=422)$.

\begin{tabular}{|c|c|}
\hline Variables & Frequency $(\%)$ \\
\hline \multicolumn{2}{|c|}{ Consumption of sweet food } \\
\hline Yes & $365(86.5)$ \\
\hline No & $57(13.5)$ \\
\hline \multicolumn{2}{|c|}{ Frequency of consumption } \\
\hline Daily & $114(31.1)$ \\
\hline $2-3^{*}$ days & $179(48.9)$ \\
\hline Once a week & $73(19.9)$ \\
\hline \multicolumn{2}{|c|}{ Bottle food childhood } \\
\hline Yes & $144(34.1)$ \\
\hline No & $278(65.9)$ \\
\hline \multicolumn{2}{|l|}{ Drink soft drink } \\
\hline Yes & $329(78.0)$ \\
\hline No & $93(22.0)$ \\
\hline \multicolumn{2}{|l|}{ Teeth brush habit } \\
\hline Yes & $165(39.1)$ \\
\hline No & $257(60.9)$ \\
\hline \multicolumn{2}{|l|}{ Frequency of bushing } \\
\hline Twice/day & $22(5.2)$ \\
\hline Once/day & $37(8.8)$ \\
\hline Sometime & $106(25.1)$ \\
\hline \multicolumn{2}{|l|}{ Material use to clean } \\
\hline Tooth brush & $67(15.9)$ \\
\hline Local chew stick & $98(23.2)$ \\
\hline \multicolumn{2}{|l|}{ Toothpaste use } \\
\hline Yes & $67(15.9)$ \\
\hline No & $355(84.1)$ \\
\hline \multicolumn{2}{|l|}{ Parent insistence } \\
\hline Yes & $124(29.4)$ \\
\hline No & $298(70.6)$ \\
\hline \multicolumn{2}{|l|}{ Dental aches } \\
\hline Yes & $169(40.0)$ \\
\hline No & $253(60.0)$ \\
\hline \multicolumn{2}{|c|}{ School health education } \\
\hline Yes & $239(56.6)$ \\
\hline No & $183(43.4)$ \\
\hline \multicolumn{2}{|l|}{ Habit mouth wash } \\
\hline Yes & $233(55.3)$ \\
\hline No & $189(44.8)$ \\
\hline
\end{tabular}

In this study, lack of oral health education program was significantly associated with dental caries. This result is similar to those studies conducted in Nepal and Bangladesh; children who did not receive oral health education were significantly associated with having dental caries [3]. This might be those children who are getting health education might have got different educational messages regarding factors contributing to dental caries and have good knowledge about how to prevent dental caries.

According to this study, lack of parent's insistence on their children for cleaning their teeth was significantly associated with dental caries. This study was supported by a study done in Mumbai (India) [22]. This might be due to the fact that the lack of parents' insistence for children may be associated with behavioral characteristics of the children like poor habit of cleaning their mouth and consuming a lot of sweet food which is associated with dental caries. 


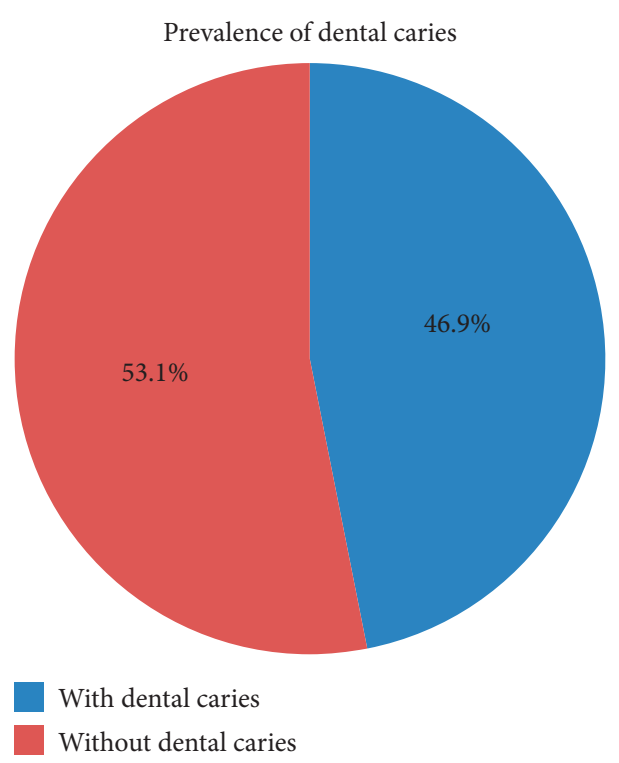

Figure 1: The prevalence of dental caries among primary school children in Alem Ketema, North Shewa, Ethiopia, 2020.

TABLe 4: Factors associated with dental caries among primary school student in Alem Ketema town, North Shewa, Ethiopia, 2020.

\begin{tabular}{|c|c|c|c|c|}
\hline Variables & Yes (\%) & No (\%) & COR (CI: $95(\%))$ & AOR (CI: 95 (\%)) \\
\hline \multicolumn{5}{|l|}{${ }^{*} \operatorname{Sex}$} \\
\hline Male & $142(57.7)$ & $104(42.3)$ & $2.926(1.950-4.390)$ & $1.975(1.067-3.656)^{*}$ \\
\hline Female & $56(31.8)$ & $120(68.2)$ & 1 & 1 \\
\hline \multicolumn{5}{|l|}{ Age } \\
\hline $7-10$ & $109(60.6)$ & $71(39.4)$ & $2.639(1.774-3.925)$ & $2.029(0.770-5.345)$ \\
\hline $11-14$ & $89(36.8)$ & $153(63.2)$ & 1 & 1 \\
\hline \multicolumn{5}{|c|}{ Grade level } \\
\hline $1-4$ & $118(59.3)$ & $81(40.7)$ & $2.604(1.757-3.860)$ & $1.352(0.508-3.597)$ \\
\hline $5-8$ & $80(35.9)$ & $143(64.1)$ & 1 & 1 \\
\hline \multicolumn{5}{|c|}{ Consumption of sweet food } \\
\hline Yes & $183(50.1)$ & $182(49.9)$ & $2.815(1.508-5.256)$ & $1.224(0.454-3.298)$ \\
\hline No & $15(26.3)$ & $42(73.7)$ & 1 & 1 \\
\hline \multicolumn{5}{|c|}{ Tooth brushing } \\
\hline Yes & $66(40.0)$ & $99(60.0)$ & 1 & 1 \\
\hline No & $132(51.4)$ & $125(48.6)$ & $1.584(1.066-2.354)$ & $1.089(0.570-2.081)$ \\
\hline \multicolumn{5}{|c|}{${ }^{*}$ Dental aches } \\
\hline Yes & $104(61.5)$ & $65(38.5)$ & $2.706(1.812-4.043)$ & $2.875(1.555-5.317)^{*}$ \\
\hline No & $94(37.2)$ & $159(62.8)$ & 1 & 1 \\
\hline \multicolumn{5}{|c|}{${ }^{*}$ Oral health education } \\
\hline Yes & $22(75.9)$ & $7(24.1)$ & 1 & 1 \\
\hline No & $97(42.0)$ & $134(58.0)$ & $4.342(1.783-10.570)$ & $4.753(1.688-13.379)^{*}$ \\
\hline \multicolumn{5}{|c|}{${ }^{*}$ Parent insistence } \\
\hline Yes & $82(66.1)$ & $42(33.9)$ & 1 & 1 \\
\hline No & $142(47.7)$ & $156(52.3)$ & $2.145(1.387-3.317)$ & $2.052(1.079-3.902)^{*}$ \\
\hline \multicolumn{5}{|c|}{${ }^{*}$ Mouth wash after food } \\
\hline Yes & $66(28.3)$ & $167(71.7)$ & 1 & 1 \\
\hline No & $132(69.8)$ & $57(30.2)$ & $5.860(3.844-8.932)$ & $4.739(2.555-8.790)^{*}$ \\
\hline
\end{tabular}

${ }^{*}$ Significance.

This study had also shown that children who were having poor mouth wash after food were 4.7 times more likely to develop dental caries. This finding is supported by a study conducted in Mumbai (India) [22] and a study done by Tamil Nadu (India) where children who had poor habit of mouth wash were of higher odds of developing dental caries [28]. Having poor wash after food may cause the accumulation of food between the teeth and create good environment for bacterial growth which causes dental caries.

5.1. Limitation of the Study. The limitation of the study was difficulty of radiological examination at a filled level which might reduce the actual magnitude of the problem. 


\section{Conclusion}

In this study, the prevalence of dental caries was higher and a common public health problem among school children. Sex, dental ache history, lack of health education, lack of parent's insistence to their children to clean their teeth, and poor practices of mouth wash after food were the factors associated with dental caries among school children.

\section{Abbreviations}

DBU: Debre Berhan University

DMFT: Decayed, Missing, and Filled Teeth

OHE: Oral health education

SPSS: Statistical Package for Social Sciences

WHO: World Health Organization.

\section{Data Availability}

Full data for this research are available from the corresponding author upon request.

\section{Ethical Approval}

Ethical clearance was obtained from Debre Berhan University College of Health Science and Department of Public Health. A letter of cooperation was written to Alem Ketema Primary School local authorities.

\section{Consent}

Written informed consent was obtained from parents and legal guardians of those students before interview and dental examination. The objective of the study was told briefly to the participants, and each volunteer was requested upon informed consent to give the request information as an interview. Only participants who agree were interviewed after their full consent and confidentiality was ensured, including not mentioning their names in any communication.

\section{Disclosure}

No funding bodies were involved in the manuscript writing, editing, approval, or decision to publish.

\section{Conflicts of Interest}

The authors declare that they have no conflicts of interest.

\section{Authors' Contributions}

All authors contributed to the design of the study and the interpretation of data.

\section{Acknowledgments}

The authors acknowledge Alem Ketema Education Office, Woreda Administration, for its cooperation and providing relevant information, and all parents and children who participated in this study.

\section{References}

[1] WHO, Sugars and Dental Caries, World Health Organisation, Geneva, Switzerland, 2017.

[2] S. Syreen, A. Anwar, G. Ahmad, and M. Z. Rahman, "Prevalence of dental caries among children residing in muslim dominated area of Laheriasarai, Darbhanga," Journal of Medical Science and Clinical Research, vol. 6, no. 12, 2018.

[3] L. P. Dixit, A. Shakya, M. Shrestha, and A. Shrestha, "Dental caries prevalence, oral health knowledge and practice among indigenous chepang school children of Nepal," BMC Oral Health, vol. 1320 pages, 2013.

[4] WHO, Oral Health, World Health Organisation, Geneva, Switzerland, 2020.

[5] K. Yadav and S. Prakash, "Dental caries: a review," Asian Journal of Biomedical and Pharmaceutical Sciences, vol. 6, pp. 1-7, 2016.

[6] S. E. Haque, M. Rahman, K. Itsuko et al., "Effect of a schoolbased oral health education in preventing untreated dental caries and increasing knowledge, attitude, and practices among adolescents in Bangladesh," BMC Oral Health, vol. 16, 2016.

[7] J. Godson, J. Csikar, and S. White, "Oral health of children in England: a call to action!" Archives of Disease in Chidhood, vol. 1, 2017.

[8] S. Ramesh and S. Sundari, "Dental caries and oral hygiene practices among school children in Chennai, India: a cross sectional study," International Journal of Contemporary Pediatrics, vol. 6, p. 2032, 2019.

[9] V. K. Chugh, K. K. Sahu, and A. Chugh, "Prevalence and risk factors for dental caries among preschool children: a crosssectional study in Eastern India," International Journal of clinical Pediatric Dentistry, vol. 11, pp. 238-243, 2018.

[10] P. D. Garkoti, R. K. Singh, C. M. S. Rawat, and S. Pandey, "Prevalence of dental caries among primary school children of Haldwani: a cross sectional study," Journal of Evolution of Medical and Dental Sciences, vol. 4, pp. 7096-7100, 2015.

[11] W. Mulu, T. Demilie, M. Yimer, K. Meshesha, and B. Abera, Dental caries and associated factors among primary school children in Bahir Dar city: a cross-sectional study," BMC Research Notes, vol. 7, p. 949, 2014.

[12] A. Amit, N. Manohar, and J. R. John, "Factors associated with dental caries in primary dentition in a non-fluoridated rural community of new South Wales, Australia," International Journal of Environmental Research and Public Health, vol. 141444 pages, 2017.

[13] D. Ozdemir, "Dental caries: the most common disease worldwide and preventive strategies," International Journal of Microbiology Research and Reviews, vol. 6, pp. 340-344, 2014.

[14] H. Y. Berhane and A. Worku, "Oral health of young adolescents in Addis Ababa-a community-based study," Open Journal of Preventive Medicine, vol. 4, pp. 640-648, 2014.

[15] B. J. Turton and C. S. Durward, "Management of early childhood caries-a comparison of different approaches," Thai Dental Public Health Journal, vol. 22, 2017.

[16] B. O. Popoola, O. O. Denloye, and O. I. Iyun, "Influence of parental socioeconomic status on caries prevalence among children seen at the university college hospital, Ibadan," Annals of Ibadan Postgraduate Medicine, vol. 11, pp. 81-86, 2013.

[17] R. Detels, M. Gulliford, Q. Abdool Karim, and C. C. Tan, Global Public Health, Oxford University Press, Oxford, UK, 2015. 
[18] A. Treerutkuarkul and K. Gruber, "Prevention is better than treatment," Bulletin of the World Health Organization, vol. 93, pp. 594-595, 2015.

[19] Federation of Wholesale Distributors, Oral Health World Wide, Federation of Wholesale Distributors, Eastbourne, UK, 2015.

[20] K. J. Chen, S. S. Gao, D. Duangthip, E. C. M. Lo, and C. H. Chu, "Early childhood caries and oral health care of Hong Kong preschool children," Clinical, Cosmetic and Investigational Dentistry, vol. 17, pp. 27-35, 2019.

[21] M. Roy, Oral Health, 2018.

[22] N. G. Pai, S. Acharya, J. Vaghela, and S. Mankar, "Prevalence and risk factors of dental caries among school children from a low socio economic locality in Mumbai, India," International Journal of Applied Dental Sciences, vol. 4, pp. 203-207, 2017.

[23] B. Arora, V. I. S. Khinda, S. Kallar, N. Bajaj, and G. S. Brar, "Prevalence and comparison of dental caries in 12 year old school going children in rural and urban areas of Ferozepur city using sic index," Dental, Oral and Craniofacial Research, vol. 1, no. 2, pp. 38-41, 2015.

[24] A. Teshome, A. Yitayeh, and M. Gizachew, "Prevalence of dental caries and associated factors among Finote Selam primary school students aged 12-20 years, Finote Selam town, Ethiopia," Oral Health and Dental Management, vol. 15, 2016.

[25] T. Zeru, L. Muruts, M. Zeru, B. Gebremariam, A. Girmay, and D. Bahre, "Prevalence of dental caries and associated factors among Aksum primary school students, Aksum town, Ethiopia 2019: a cross-sectional," Journal of Dental and Oral Health, vol. 6, 2019.

[26] N. Elfaki, A. S. Elgarrai, A. I. Shwail, S. L. Brair, and M. A. Alsheikh, "Prevalence of dental caries among primary school attendees in najran-Saudi Arabia," IOSR Journal of Dental and Medical Sciences, vol. 13, no. 11, pp. 37-41, 2014.

[27] A. K. Andegiorgish, B. W. Weldemariam, M. M. Kifle1 et al., "Prevalence of dental caries and associated factors among 12 years old students in Eritrea," BMC Oral Health, vol. 17169 pages, 2017.

[28] G. Parasuraman, Y. G. Krishna, M. Kaviya, N. A. Jain, P. Rajendiran, and R. Dutta, "A study on the prevalence of dental caries among the school-going children in Tamil Nadu," International Journal of Community Medicine and Public Health, vol. 4, 2017.

[29] L. G. T. Martins, K. C. R. Pereira, S. X. S. Costa et al., "Impact of dental caries on quality of life of school children," Brazilian Research in Pediatric Dentistry and Integrated Clinic, vol. 16, pp. 307-312, 2016.

[30] C. A. Akinyamoju, D. M. Dairo, I. A. Adeoye, and A. O. Akinyamoju, "Dental caries and oral hygiene status: survey of school children in rural communities, Southwest Nigeria," Nigerian Postgraduate Medical Journal, vol. 25, pp. 239-245, 2019.

[31] M. B. Hatkehlouei, H. Tari, A. H. Goudarzian, and H. Hali, "Decayed, missing, and filled teeth (DMFT) index among first-grade elementary students in Mazandaran Province, Northern Iran," International Journal of Pediatrics, vol. 5, pp. 5069-5077, 2017.

[32] P. Goenka, S. Dutta, N. Marwah, A. Sarawgi, M. Nirwan, and P. Mishra, "Prevalence of dental caries in children of age 5 to 13 Years in district of vaishali, Bihar, India," International Journal of Clinical Pediatric Dentistry, vol. 11, no. 5, pp. 359-364, 2018.

[33] M. Ali Youssefi and S. Afroughi, "Prevalence and associated factors of dental caries in primary schoolchildren: an Iranian setting," International Journal of Dentistry, vol. 2020, Article ID 8731486, 7 pages, 2020.

[34] M. Ferraro and A. R. Vieira, "Explaining gender differences in caries: a multifactorial approach to a multifactorial disease," International Journal of Dentistry, vol. 2010, Article ID 649643, 5 pages, 2010. 